\title{
Pre-radiotherapy PSA Level as A Predictor
}

\author{
Viroj Wiwanitkit
}

\section{Respected Editor,}

$\mathrm{T}^{\mathrm{h}}$ The recent publication on "pre-radiotherapy PSA level as a predictor" is very interesting. ${ }^{[1]}$ Tsan et al., concluded that "in patients with increasing prostate-specific antigen (PSA) levels following radical prostatectomy (RP), local radiotherapy (RT) should be administered prior to biochemical failure (PSA $\geq 0.2$ ) to ensure good biochemical progression - free survival (bPFS). ${ }^{[1]}$ " It seems that the PSA level determination can be useful in predicting and planning for the management of prostate cancer. Nevertheless, there are many concerns on this work. First, although PSA seems to be a good cancer biomarker for prostate cancer, increased level can be seen in other prostatic disorders such as chronic prostatitis. ${ }^{[2]}$ In case there are concurrent disorders, the PSA level might be altered and is not reliable. Second, ensuring the quality control of PSA measurement is very important. Errors in laboratory investigation can be expected, and this can be a problem in actual clinical practice. In a certified laboratory, high rate of pre-analytic error is still being reported.$^{[3]}$ Biases of presently used PSA test are reported, and this must be kept in mind by all practitioners. ${ }^{[4]}$

\section{REFERENCES}

1. Tsan DL, Fan KH, Chen YC, Chuang CK, Lee CC, Hong JH. Pre-radiotherapy PSA level as a predictor for biochemical control in prostate cancer patients receiving radiotherapy after radical prostatectomy. Biomed J 2013;36:71-6.

2. Sindhwani P, Wilson CM. Prostatitis and serum prostate-specific antigen. Curr Urol Rep 2005;6:307-12.

3. Wiwanitkit V. Types and frequency of preanalytical mistakes in the first Thai ISO 9002:1994 certified clinical laboratory, a 6 - month monitoring. BMC Clin Pathol 2001;1:5.

4. Roddam AW, Rimmer J, Nickerson C, Ward AM. NHS Prostate Cancer Risk Management Programme. Prostate-specific antigen: Bias and molarity of commercial assays for PSA in use in England. Ann Clin Biochem 2006;43:35-48.

From the Hainan Medical University, China; Faculty of Medicine, University of Nis, Serbia, Thailand

Received: Apr. 23, 2013; Accepted: May 23, 2013

Correspondence to: Prof. Viroj Wiwanitkit, Faculty of Medicine, University of Nis, Serbia, Thailand. Wiwanitkit House, Bangkhae, Bangkok, Thailand. Tel: 66-24132436; Fax: 66-24132436; E-mail: wviroj@yahoo.com

DOI: $10.4103 / 2319-4170.113859$ 